\title{
Thermofixieren von Seilen aus hochfesten Synthesefasern
}

\author{
DIPL.-ING. THORSTEN HEINZE, DIPL.-ING. JENS MAMMITZSCH \\ INSTITUT FÜR FÖRDERTECHNIK UND KUNSTSTOFFE, PROFESSUR FÖRDERTECHNIK, TU CHEMNITZ
}

\begin{abstract}
Zusammenfassung
\end{abstract}
Seile aus hochfesten, synthetischen Fasern erlangen in den letzten Jahren immer stärkere Bedeutung. Insbesondere beim Abspannen, Heben und Fördern von Lasten über große Längen bringen sie aufgrund des geringen Tragmittelgewichtes und der mit Stahl vergleichbaren Festigkeit entscheidende Vorteile mit. Dennoch wird durch die machartbedingte Lage der Synthesefasern die Festigkeit des Seiles reduziert und die für technische Anwendungen schädliche Strukturdehnung erhöht. Das Thermofixieren stellt eine Möglichkeit bereit, die Molekülketten der Fasern neu zu orientieren, den Kraftfluss zu homogenisieren und Dehnungseffekte zu reduzieren. Um die technischen Möglichkeiten, Potentiale und Risiken des Thermofixierens von Faserseilen sowie die resultierenden Auswirkungen auf das Biegewechsel- bzw. Kriechverhalten zu untersuchen, wurden am Institut für Fördertechnik und Kunststoffe der Technischen Universität Chemnitz umfangreiche Versuche an Garnen und Seilen aus industriell verfügbaren, hochfesten Polymerfasern durchgeführt.

\begin{abstract}
Ropes made from synthetic high-strength fibers became more and more demanded, during the last years. Especially in hoisting and conveying applications, they can be applied with great benefit. For many of these applications, the strength of the ropes is a main decision criterion. To point out the potentialities and risks of thermosetting of fiber ropes, the Institute of Materials Handling, Conveying and Plastics Engineering of Chemnitz University of Technology has done wide-ranging tests on yarns and ropes, made of industrially available high-strength polymer fibers, to analyze the technical capabilities of thermosetting for increasing the strength of fiber ropes and determine the resulting effects to bending fatigue.
\end{abstract}

\section{Motivation}

Die Zugfestigkeit textiler Seile wird durch die anisotropen Eigenschaften der Fasern und deren machartbedingter Lage zur Zugrichtung teils erheblich herabgesetzt. Darüber hinaus bewirkt die Seilmachart eine hohe Strukturdehnung und niedrige Seildichte. Die Strukturdehnung, die selbst bei hochfesten Seilgeflechten je nach Maschinenkonfiguration bis zu 6\% im Arbeitsbereich (30\% MBL) betragen kann, führt insbesondere bei großen Anschlag- bzw. Förderlängen zu unzulässigen Längenänderungen an der Anlage und die hohe Querkontraktion zu veränderlichen Auflageverhältnissen in der Seilscheibe sowie verminderter Formstabilität, die wiederum die Lebensdauer reduzieren.

Das Thermofixieren von technischen Textilien hat sich vor allem bei der Fertigung und Veredlung von Bändern und Gurten etabliert, um gewisse Eigenschaften der Enderzeugnisse positiv zu beeinflussen. So wird das Verfahren bereits in der Serienfertigung eingesetzt, um bei textilen Erzeugnissen aus Polyamid die elastische Dehnung und die Kriechneigung zu reduzieren oder bei diversen Polyester-Typen den Effekt des Schrumpfens bei Wärmezufuhr zu unterdrücken.

Auch bei den hochfesten Fasern ist das Verfahren bereits in einigen Spezialanwendungen im Einsatz [Gle2005], um bei Seilen aus hochfesten Polyethylenfasern (Dyneema, Spectra) die Festigkeit zu erhöhen und gleichzeitig Dehnung und Kriechneigung zu verringern. Die Motivation zu den nachfolgend aufgeführten Untersuchungen ergibt sich aus der Tatsache, dass bis jetzt keine gesicherten Erkenntnisse über den Einfluss der Fixierparameter der Polyethylen-Fasern vorliegen. Einige Seilhersteller produzieren zwar hochfeste Polyethylen-Seile, die einem Thermofixierprozess unterzogen worden sind, die zugehörigen Prozessparameter sind jedoch meist empirisch ermittelt und basieren auf internen unveröffentlichten Versuchen.

Darüber hinaus wurde bisher nicht untersucht, ob auch andere hochfeste Polymerfasern, wie die hochtemperaturbeständigen Aramide und TLCP's, die Möglichkeit bieten, durch den Thermofixierprozess Eigenschaften wie Festigkeit und Dehnung positiv zu beeinflussen. 


\section{Grundlagen des Thermofixierens}

Das Thermofixieren ist ein Verfahren zur thermo-mechanischen Behandlung von textilen Gebilden aus Polymerfasern. Dazu wird die Textilie auf eine werkstoffabhängige Temperatur erwärmt und anschließend mit einer Kraft beaufschlagt. In der Textilindustrie wird dies für flächige Textilien meist mit einem Spannrahmen und Walzen zur Erzeugung einer Längsspannung realisiert. Das Erwärmen des Bauteils kann dabei mittels Heißwasser, Wasserdampf oder Heißluft erfolgen [Nen2006]. Die Höhe der Kraft ist von der Festigkeit des Polymerwerkstoffes und die Durchwärmzeit von der Geometrie und Wärmeleitung des textilen Gebildes abhängig. In der Industrie wird aus Erfahrungswerten angestrebt, nach der vollständigen Durchwärmung eine Fixierzeit von mindestens drei Minuten zu gewährleisten. Nach Abschluss der Behandlung wird die Textilie unter Last abgekühlt.

Das Thermofixeren wird in der Textilindustrie häufig zur Behandlung von Textilien aus Naturfasern oder Synthesefasern wie Polyester- und Polyamidwerkstoffen eingesetzt, um das bei diesen Werkstoffen auftretende Schrumpfen unter Wärmeeinwirkung zu unterbinden und so eine gute Formbeständigkeit bei hohen Einsatztemperaturen zu gewährleisten [Top1977].

Die Dehnung textiler Tragmittel oder Bauteile setzt sich aus der Strukturdehnung und der werkstoffspezifischen Dehnung zusammen, die beide zeit- und temperaturabhängiges Verhalten hervorrufen. Als auftretender Nebeneffekt des Thermofixierens können diese visko-elastischen Effekte zum Teil erheblich reduziert werden. Durch das Belasten bei einer Temperatur, die oberhalb der Glasübergangstemperatur liegt, werden die amorphen Bereiche, die in jedem teilkristallinen Polymer vorhanden sind, gestreckt und dabei in Richtung der Belastungsvekoren des textilen Gebildes orientiert [Sch2006]. Ist das Verstrecken der Fasern bereits ausgereizt, d.h. sind die Molekülketten in Faserrichtung ausgerichtet, ist eine Umorientierung der Moleküle in den Ersatzmaterialien wie Garnen, Seilsträngen und dem Seil selbst, noch immer möglich. Dies bedeutet primär eine Ausrichtung in Zugrichtung des Bauteils, aber auch in transversaler Richtung durch Flächenpressungen an den Überkreuzungspunkten der Litzen und den Walzen des Reckwerkes.

Versuche an der TU Chemnitz haben gezeigt, dass das Thermofixieren von Garnen im Anlieferungszustand (außer Vectran $\left.{ }^{\circledR}\right)$ meist keine signifikante Steigerung der Festigkeit erzielt, während bei verzwirnten Garnen gleichen Titers unter vergleichbaren Bedingungen eine signifikante Steigerung der Zugfestigkeit erreicht werden kann. Dieser Effekt ist vermutlich dadurch zu erklären, dass die Kristallite der Fasern bereits maximal orientiert und damit auch keine Festigkeitssteigerungen paralleler Faserbündel möglich sind.

Beim Verzwirnen werden die Fasern miteinander verdreht, ähnlich wie bei geschlagenen Seilen. Dadurch orientieren sich die Faserachsen schraubenförmig um die Zwirnachse. Durch die Umschlingungsreibung tragen bei Anlegen einer äußeren Kraft alle Fasern nahezu gleichmäßig. Die durch das Verdrehen eingetragenen Querkräfte führen jedoch zu einer über den Geometriefaktor $\operatorname{Cos}^{2} \alpha$ [Kil1964] hinaus abnehmenden Festigkeit des Zwirns, da die Fasern erheblich niedrigere transversale Festigkeits-und Steifigkeitswerte aufweisen als in axialer Richtung. Wird ein solches gezwirntes Garn dem Thermofixierprozess unterzogen, so werden sich die Kristallite während des Prozesses umorientieren, sodass sich die Hauptorientierungsrichtung zur Garnachse hin verschiebt. Dieser Vorgang wird sich mit dem Verstrecken der amorphen Bereiche überlagern. Aufgrund der Tatsache, dass alle Fasern nahezu gleichmäßig tragen, werden auch alle Fasern gleichmäßig verstreckt und fixiert, was dann zu einer nutzbaren Festigkeitserhöhung führt.

Die Litzen in einem geflochtenen Seil bestehen aus mehreren Garnen, die zu einer Litze verzwirnt werden. Auch hier tragen alle Fasern gleichmäßig und auch hier findet mit zunehmendem Flechtwinkel eine Festigkeitsminderung statt. Abbildung 1 zeigt die Festigkeitsabnahme geflochtener Seile mit zunehmendem Flechtwinkel. Beim Thermofixieren derartiger Seile lässt sich genau wie bei den Garnen die Festigkeit in nutzbarem Umfang verbessern. Die Höhe des Festigkeitszuwachses ist dabei vom Verhältnis der applizierten Kraft zur Seilkonstruktion abhängig (Anzahl der Garne in einer Litze, Anzahl der Drehungen der Litze, Anzahl und Titer der Litzen, Flechtlänge, Seildurchmesser). Für den technischen Gebrauch bedeutsamer dürfte jedoch die wesentlich geringere Strukturdehnung gegenüber dem Originalseil sein, da Schwierigkeiten bei der Montage aber auch während des Betriebes durch teils undefinierte Anfangsdehnungen vermindert werden. Darüber hinaus begünstigt sie, die für technische Anwendungen notwendigen Veredlungsprozesse, wie die Mantelextrusion, da die Dehnung von Matrix und Seil aufeinander abgestimmt und Schäden der Matrix durch eine zu hohe Strukturdehnung vermieden werden können. 


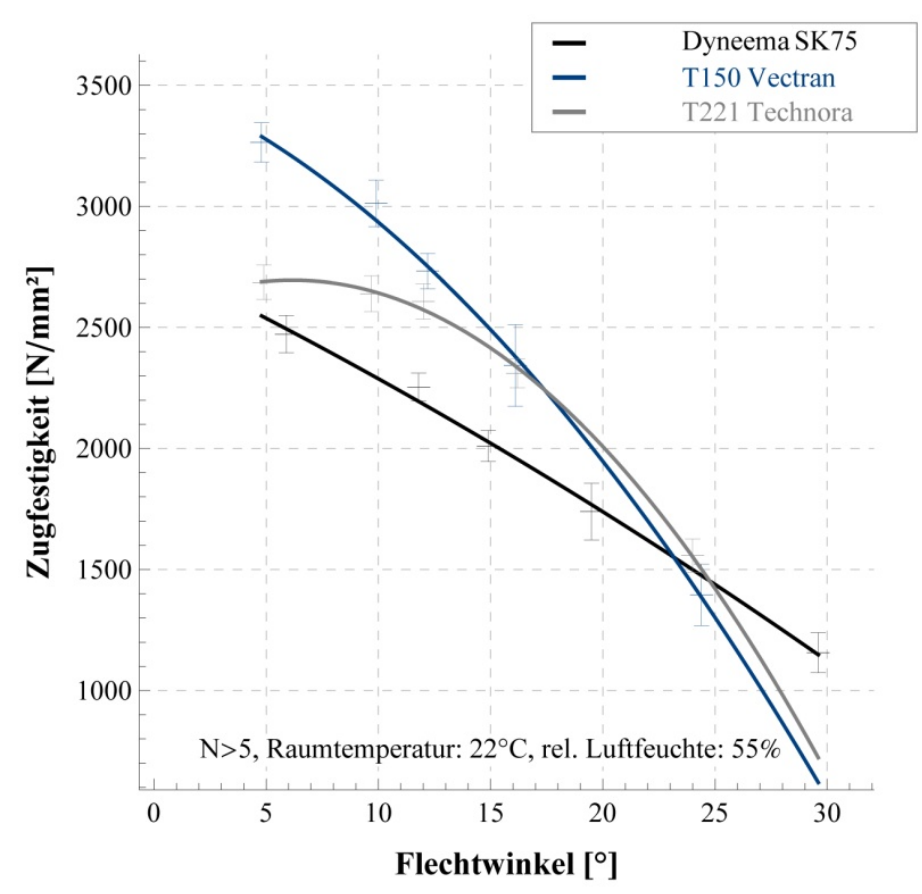

Abbildung 1: Festigkeitsabnahme mit zunehmendem Flechtwinkel (2mm-Seile)

\section{DSC-Analyse}

Die DSC-Analyse (Differential Scanning Calorimetry = Dynamische Differenzkalorimetrie) ist ein Verfahren zur thermischen Analyse von polymeren Werkstoffen. Bei der Analyse, die nur wenige Milligramm des Probenmaterials benötigt, werden ein Behälter mit der Probe und ein Referenzbehälter ohne Probe definierten Temperaturänderungen ausgesetzt. Üblicherweise erfolgt ein 1. Aufheizprozess, anschließend ein definiertes Abkühlen und abschließend ein 2. Aufheizen. Dabei wird die Geschwindigkeit der Temperaturänderung vorgegeben, z.B. $10 \mathrm{~K} / \mathrm{min}$. Durch im Werkstoff entstehende Reaktionen kommt es zu Temperaturunterschieden gegenüber der Referenzkammer, die auf den Wärmefluss in der Probe schließen lassen. Der auf die Probenmasse bezogene Wärmefluss wird in einem Diagramm über der Temperatur angetragen. Im Diagramm sind Peaks erkennbar, die anhand ihrer Position und Höhe bestimmten Werkstoffvorgängen zugeordnet werden können. Daher wird das Verfahren unter anderem zur Detektion endothermer Vorgänge (Schmelzen, Glasübergang) bzw. exothermer Vorgänge (Kristallisation, Zersetzen) aber auch zur Ermittlung der Wärmekapazität von Kunststoffen verwendet [Ehr2003].

Aufgrund der Ausbildung von Kristalliten lassen sich teilkristalline Kunststoffe nicht nur über den Glasübergangspunkt (Glasübergangstemperatur) sondern auch über die Ausprägung der KristallitSchmelztemperatur eindeutig identifizieren. Die Kristallitschmelztemperatur gibt die Temperatur an, bei der die Kristallite in einem teilkristallinen Werkstoff beginnen, ihre Anordnung aufzulösen. Die Polymerketten nehmen dann eine Struktur ähnlich den amorphen Bereichen an. Dieser Vorgang setzt bei den meisten Kunststoffen kurz vor dem eigentlichen Beginn der Schmelze ein.

Aus einer DSC-Kurve lassen sich aber auch reversible und irreversible Effekte in der Phasenstruktur von Kunststoffen ermitteln. Vergleicht man beispielsweise die Kurven des ersten und zweiten Aufheizvorganges, so wird man bei einigen Kunststoffen, z.B. Dyneema ${ }^{\circledR}$ von DSM, feststellen, dass sich die Peaks in ihrer Lage und Ausprägung unterscheiden.

In Abbildung 2 ist eine an der TU Chemnitz ermittelte DSC-Kurve für Dyneema ${ }^{\circledR}$ SK75 dargestellt. Betrachtet man in der Kurve des 1 . Aufheizens den Peak bei $148^{\circ} \mathrm{C}$ und vergleicht diesen mit dem Peak bei $116^{\circ} \mathrm{C}$ in der Abkühlkurve, kann man daraus erkennen, dass der Peak bei $148^{\circ} \mathrm{C}$ die Temperatur der Kristallitschmelze für Dyneema ${ }^{\circledR}$ SK75 im Anlieferungszustand beschreibt. Weiterhin ist ersichtlich, dass die Rekristallisation erst bei niedrigeren Temperaturen (etwa $116^{\circ} \mathrm{C}$ ) abgeschlossen ist. Bezieht man nun die Kurve des zweiten Aufheizens in diese Betrachtungen ein, erkennt man, dass die Temperatur der Kristallitschmelze sich nach dem 1. Aufheizen und anschließenden Abkühlen in Richtung niedrigerer Temperaturen $\left(137^{\circ} \mathrm{C}\right)$ verschoben hat. Wenn man den Werkstoff nach dem 2. Aufheizen abkühlt und ein drittes Mal aufheizt, so unterscheidet sich die Kurve des dritten Aufheizens nicht von der des 2. Aufheizens. Man erkennt also, dass das Erschmelzen der Kristallite und die Rekristallisation grundsätzlich reversible Vorgänge sind, was bei thermoplastischen Kunststoffen eine bekannte Tatsache ist. 


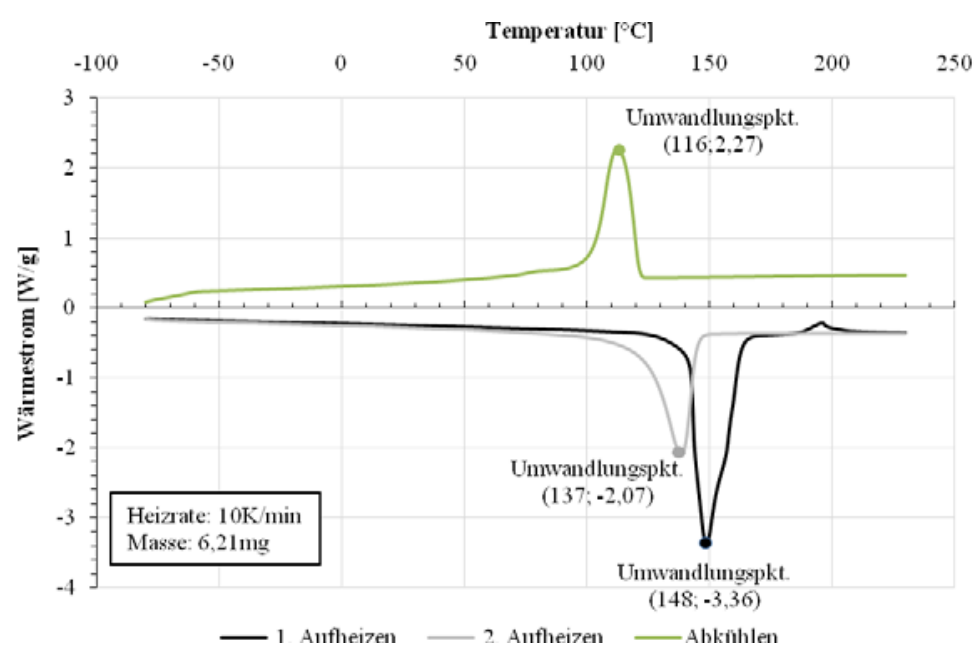

Abbildung 2: DSC-Kurve Dyneema ${ }^{\circledR}$ SK75-Probe

Weiterhin ist aus der Verschiebung der Peaks erkennbar, dass die Kristallite im Anlieferungszustand sich von den Kristalliten nach der Rekristallisation unterscheiden. Das wird besonders aus zwei Fakten deutlich, zum einen setzt die Kristallitschmelze im Anlieferungszustand erst bei deutlich höheren Temperaturen ein als beim 2. Aufheizvorgang, zum Anderen ist die Amplitude des Peaks beim 1. Aufheizen deutlich höher als beim 2. Aufheizen.

Dieser Unterschied lässt sich aus der Herstellung erklären. Dyneema® SK75 ist ein hochfester, hochmoduliger Polyethylen-Faserwerkstoff, der durch ein Gel-Spinnverfahren hergestellt wird. Der Polyethylen-Werkstoff wird in einem Lösungsmittel zu einer gelartigen Masse gequollen und mit hohen Abzugsgeschwindigkeiten durch Spinndüsen abgezogen. Dadurch kommt es zu einer sehr hohen Orientierung der Kristallite entlang der Faserachse, die den höheren Energieaufwand für das Erreichen der Kristallitschmelze erklärt. Durch das Schmelzen der Kristallite und anschließendes Abkühlen ohne Last während der DSC-Analyse, geht die hohe Orientierung der Kristallite verloren, da während des gesteuerten Abkühlens keine Scherkräfte in der Schmelze wirken. Die Kristallite können sich beliebig orientieren, was zu einer veränderten Phasenstruktur führt. Deshalb ist bei erneutem Aufheizen der Peak der Kristallitschmelze verschoben.

Für andere teilkristalline Polymerwerkstoffe lassen sich gleichartige Kurven ermitteln. Dabei ist auch dort, je nach Kristallisationsgrad und Orientierung der Kristallite, ein ähnliches Auftreten von Peak-Verschiebungen oder sogar das „Auslöschen“ von Peaks erkennbar.

Abbildung 3 und Abbildung 4 zeigen die DSC-Kurven von Vectran ${ }^{\circledR}$ und Technora ${ }^{\circledR}$, die ebenfalls an der TU Chemnitz ermittelt worden sind. Bei Vectran ${ }^{\circledR}$ handelt es sich um einen aromatischen Polyester-Faserwerkstoff, der ebenfralls durch seine Herstellung (Schmelzspinnverfahren) eine extrem hohe Orientierung der Kristallite entlang der Faserachse aufweist. Vectran ${ }^{\circledR}$ zeigt beim Vergleich der ersten und zweiten Aufheizkurven keine Verschiebungen von Peaks, jedoch ist eindeutig erkennbar, dass die Peaks oberhalb von $230^{\circ} \mathrm{C}$ ausgelöscht werden.

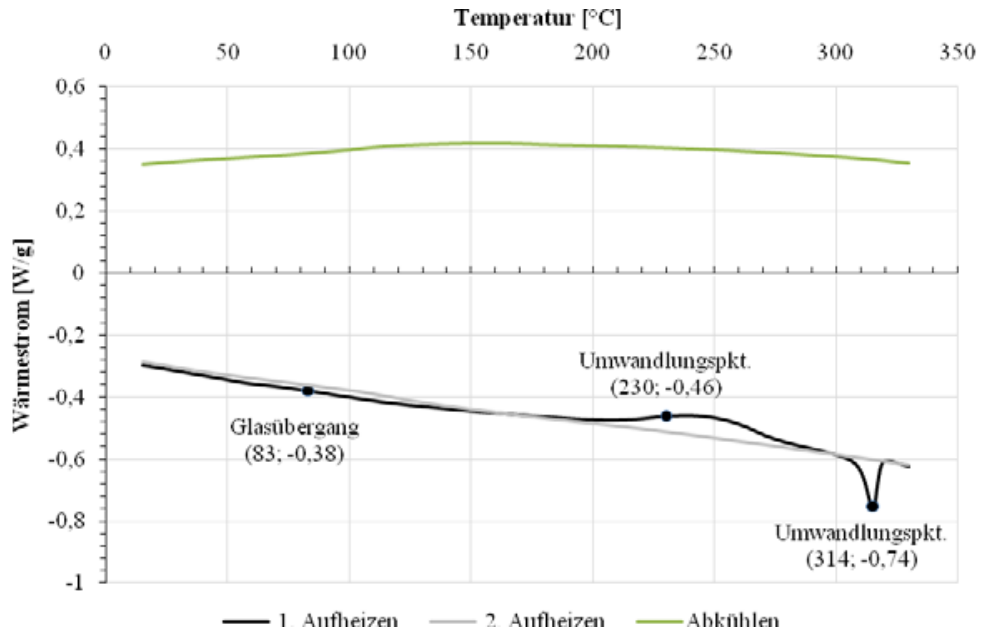

Abbildung 3: DSC-Kurve Vectran ${ }^{\circledR}$-Probe 
Das zeigt, dass dort vollständig irreversible Veränderungen in der Phasenstruktur auftreten. Die Peaks oberhalb von $314^{\circ} \mathrm{C}$ geben dabei nicht die Kristallitschmelztemperatur an. Vectran ist ein sogenanntes TLCP (thermotrope liquid crystal polymer), bei welchem auch in der Schmelze kristallitartige Strukturen in den Polymerketten vorhanden sind. Da also keine echte Kristallitschmelze auftritt, können die Peaks oberhalb von $330^{\circ} \mathrm{C}$ (so gibt der Hersteller die Schmelztemperatur an) nur Umwandlungsvorgänge derart zeigen, dass die Orientierung der Kristallite in der Schmelze verloren geht. Beim Abkühlen erfolgt dann eine zufällige Anordnung der Kristallite. Dadurch erklärt sich auch der Festigkeitsverlust der Faser bei Temperaturen oberhalb von $230^{\circ} \mathrm{C}$.

Die DSC-Kurve von Technora ${ }^{\circledR}$ zeigt bei $104^{\circ} \mathrm{C}$ einen kleinen Peak, der in der zweiten Aufheizphase nicht mehr vorhanden ist. Da der Peak sehr nahe an der Glasübergangstemperatur liegt, ist davon auszugehen, dass es sich nicht um einen Kristallitschmelzpunkt handelt. Der Glasübergangspunkt bezeichnet im Allgemeinen die Temperatur, bei der sich das Werkstoffverhalten von hart-spröd zu visko-elastisch ändert.

Technora ${ }^{\circledR}$ ist ein teilkristalliner, aromatischer Polyamidwerkstoff, der keine Schmelztemperatur zeigt, sich jedoch bei ca. $550^{\circ} \mathrm{C}$ zersetzt. Auch hier bedeutet das Verschwinden des Peaks, dass nicht umkehrbare Gefügeveränderungen, vermutlich eine Änderung der Orientierung, aufgetreten sind.

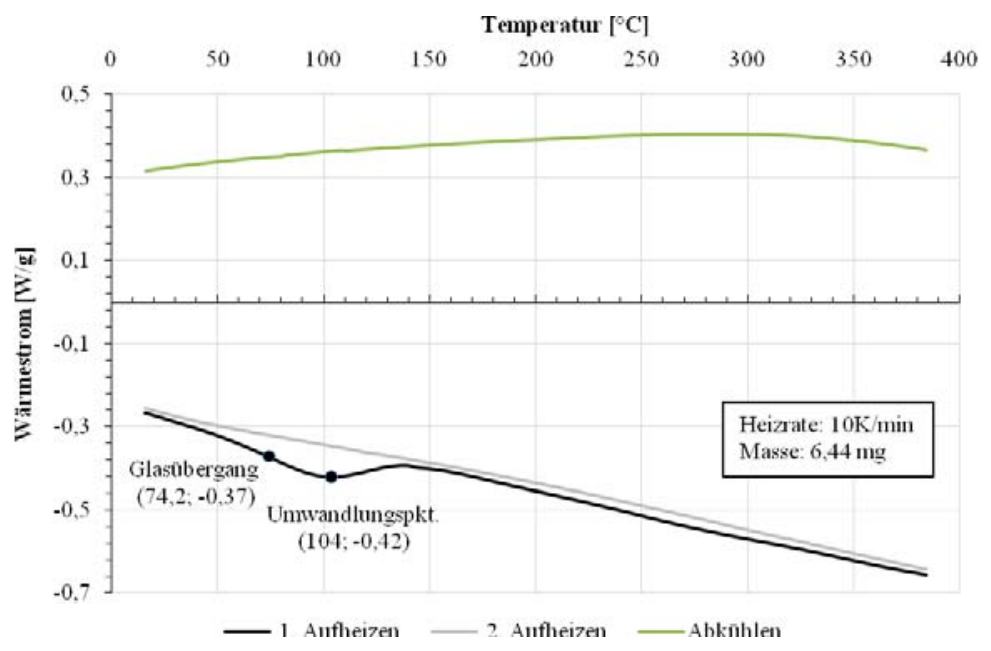

Abbildung 4: DSC-Kurve Technora ${ }^{\circledR}$-Probe

Die Thermofixierung kann nur in einem Temperaturbereich funktionieren, in dem es möglich ist, die Struktur der Polymerketten zu verändern und zu fixieren. Dabei ist es zweckmäßig, im Bereich der beginnenden Kristallitschmelze zu arbeiten. Die DSC-Kurven eignen sich gut, um für einen Faserwerkstoff genau diese Temperaturen zu ermitteln.

Durch die Faserherstellung kommt es vor allem bei Polyesterwerkstoffen zur Ausbildung von metastabilen Kristalliten. Diese lösen sich zu Beginn der Kristalliterschmelzung zuerst auf und bilden sich in amorphe Bereiche um. Dies bewirkt zum Beispiel bei aliphatischen Polyester-Werkstoffen die hohe Schrumpfung unter Temperatureinwirkung.

\section{Versuchsaufbau}

Die Anlage, auf der die Versuche durchgeführt wurden, ermöglicht neben dem Thermofixieren auch das Beschichten und Imprägnieren von Seilen aus Synthesefasern. Der prinzipielle Aufbau der Anlage ist in Abbildung 5 dargestellt. Für das Thermofixieren sind die Halte-Reckwerke sowie der Warmluft-Heiztunnel mit angeschlossener Kühlzone wichtig. Über die Halte-Reckwerke wird die Fixierkraft in das Seil eingeleitetund der Vorschub realisiert. Um die Kraft langsam aufzubauen wird mit mehrfachen Umschlingungen um gravierte Walzen gearbeitet. Die Anlage lässt sich entweder weggeregelt, indem die Geschwindigkeit des zweiten HalteReckwerkes z.B. konstant um 10\% höher ist als die des Ersten, oder kraftgeregelt betreiben. Das Thermofixieren in kraftgeregelter Verfahrensweise ist dabei in Hinblick auf reproduzierbare Ergebnisse besser geeignet. Da durch die geringen Reibwerte der meisten Kunststoffe gegen Stahl, Schlupf auf den Walzen auftritt, wird dieser bei drehzahlgeregeltem Verfahren nicht ausgeglichen. Bei kraftgeregeltem Verfahren wird eine konstante Kraft vorgegeben. Sollte kurzzeitig Schlupf auftreten, so wird der auftretende Kraftabfall von der Maschine erkannt und durch erhöhen der Drehzahlen am Auslauf und/oder verringern der Drehzahlen am Einlauf kompensiert. 


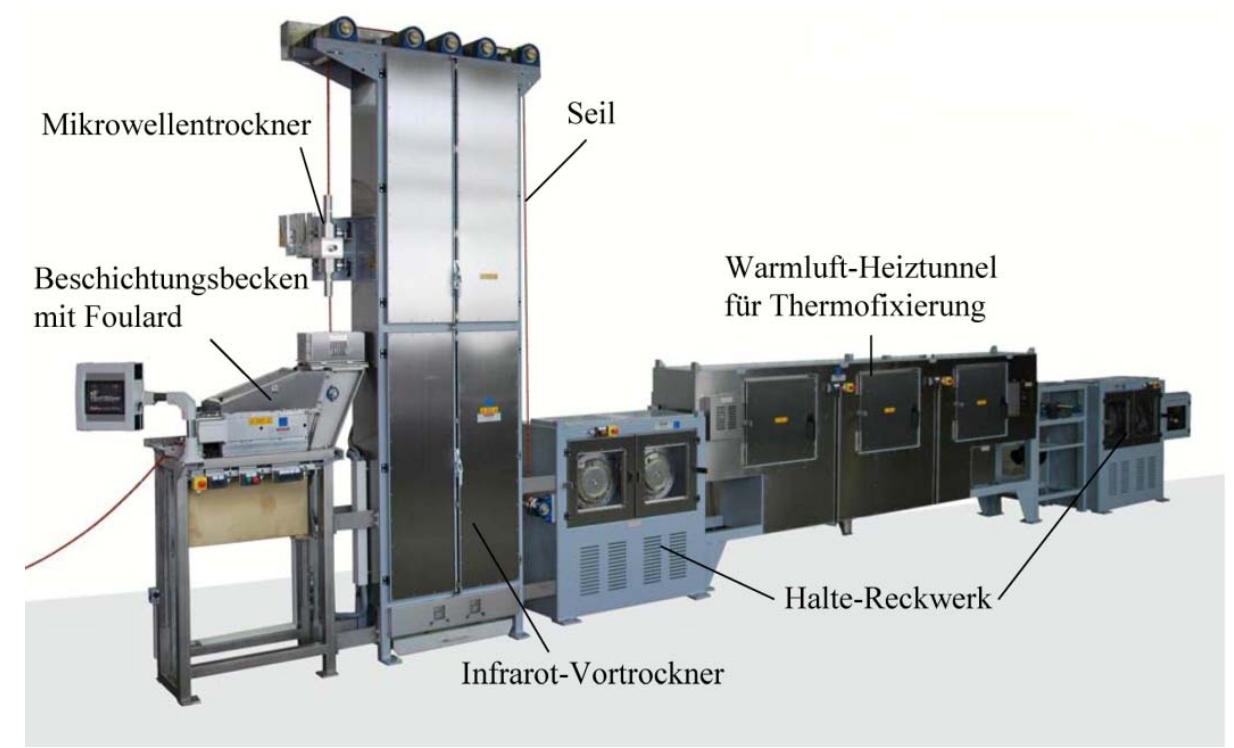

Abbildung 5: Seilfixieranlage (RopeLiner, Firma Mageba Textilmaschinen GmbH\&Co.KG)

Der Warmluft-Tunnel dient zum Erzeugen und aufrecht erhalten einer stabilen Temperatur. Dadurch soll gewährleistet werden, dass jeder Bereich des Seiles nach dem Durchlaufen des Tunnels auf die gleiche Temperatur erwärmt wird, um unterschiedlich fixierte Bereiche zu vermeiden.

\section{Ergebnisse der Thermofixierung}

Zur Abklärung der Thermofixierbarkeit und zur Variation der Prozessparameter (Fixierspannung und Einwirkdauer) sind zunächst unverdrehte und verdrehte Garne aus Dyneema ${ }^{\circledR}$, Vectran ${ }^{\circledR}$ und Technora ${ }^{\circledR}$ verwendet worden. Die Fixiertemperaturen im Heiztunnel wurden aus den DSC-Kurven ermittelt und betragen für Dyneema ${ }^{\circledR} S K 75125^{\circ} \mathrm{C}$, für Vectran ${ }^{\circledR} 230^{\circ} \mathrm{C}$ und für Technora ${ }^{\circledR} 100^{\circ} \mathrm{C}$. Die Garne wurden unter einer leichten Vorspannung in den Heiztunnel eingelegt und nach Erreichen der Fixiertemperatur mit Massestücken von $0,1 \mathrm{~kg} ; 05 \mathrm{~kg} ; 1 \mathrm{~kg} ; 2 \mathrm{~kg}$; $5 \mathrm{~kg}$ und $10 \mathrm{~kg}$ belastet. Das Reckwerk wurde wegen der Messungenauigkeit bei diesen Kräften nicht verwendet. Die Variation der Einwirkdauer von 1min; 3min; 6min und 9min bestätigte eine optimale Einwirkzeit von 3min. Diese Zeit beginnt jedoch erst, wenn das textile Gebilde vollständig durchwärmt ist. Dies ist vor allem bei dicken Seilen zu beachten, da diese wegen der niedrigen Wärmeleitfähigkeit des Grundmaterials einige Zeit benötigen, bis sich im Kern die gleiche Temperatur einstellt wie außen. Wird der Fixierprozeß zu zeitig begonnen bzw. zu zeitig beendet, werden die äußeren und inneren Filamente unterschiedlich verstreckt, was einen inhomogenen Kraftfluss und geringe Seilfestigkeiten zur Folge hat.

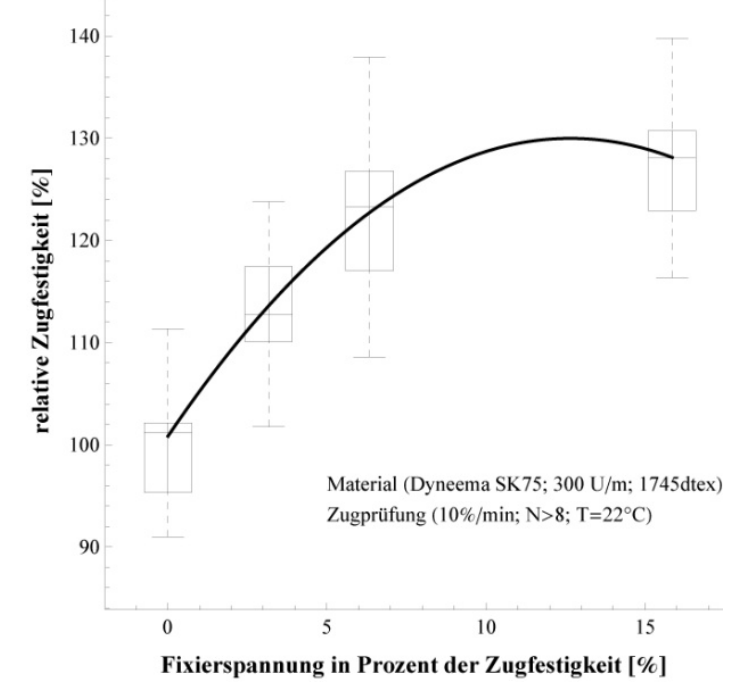

Abbildung 6: Thermofixierung von verdrehtem Dyneema ${ }^{\circledR}-G a r n$ 
Das Dyneema®-Garn (HMPE) ließ sich gut fixieren. Die Zugfestigkeit konnte bei stark verdrehten Garn $(300 \mathrm{U} / \mathrm{m})$ wie in Abbildung 6 dargestellt deutlich erhöht werden, während bei unverdrehtem Garn keine Festigkeitszuwächse beobachtet wurden.

Das Optimum der Fixierspannung von Dyneema ${ }^{\circledR}$-Garn liegt zwischen $10 \%<\sigma_{\mathrm{F}} / \mathrm{R}_{\mathrm{Z}}<15 \%$. Ein Belasten über $15 \%$ hinaus, führte zu einer Festigkeitsabnahme bis hin zu einem Zerreißen bei 32\%. Darüber hinaus konnte bereits bei $15 \%$ eine starke Plastifizierung und Verschmelzung der Fasern beobachtet werden. Dieser stabförmige Zustand führt insbesondere beim Biegen über Scheiben zu Schäden am Seil, die zwingend vermieden werden müssen. So ist auch hier ein Kompromiss zwischen maximaler Zugfestigkeit und gutem Biegeverhalten anzustreben.

Anders als bei den TLCP- und Aramid-Fasern, die durch ihre aromatischen Ringbindungen sehr große Blockkräfte aufweisen, ist bei den HMPE-Fasern ein Abgleiten von Molekülketten unter hohen Temperaturen sehr leicht möglich. Dies führt beim Fixierprozess zu einer starken Längenänderung des Garnes. So konnte in einer sich anschließenden Messung der Fasern eine nicht unerhebliche Verringerung von Garnfeinheit und Verdrehung festgestellt werden. Die Ergebnisse hierzu sind in Abbildung 7 dargestellt. Kommt ein Thermofixierprozess bei den Dyneema-Seilen zur Anwendung, ist die Verringerung der Seilfeinheit und damit des tragenden Querschnittes bei der Dimensionierung zu berücksichtigen.
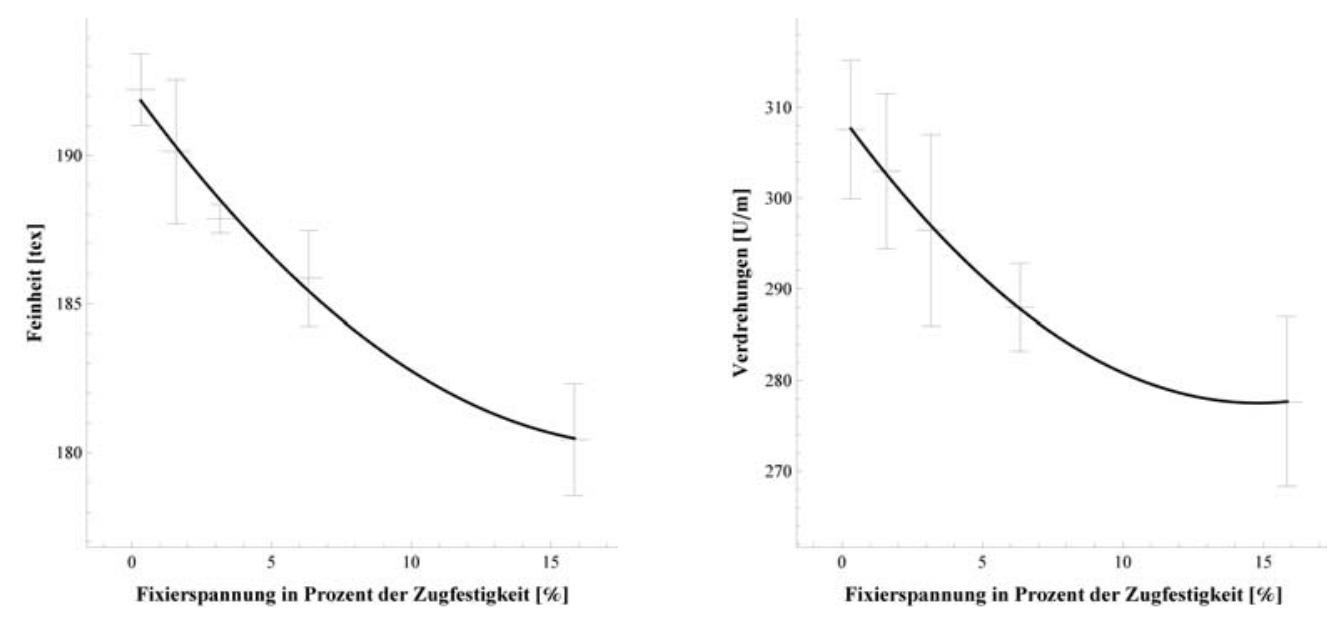

Abbildung 7: Abnahme der Garnfeinheit und Garndrehung

Entgegen verbreiteten anderen Auffassungen, konnte die Thermofixierbarkeit hochfester Hochtemperatur-Fasern wie TLCP (Vectran $\left.{ }^{\circledR}\right)$ und den Aramiden (Technora $\left.{ }^{\circledR}\right)$ nachgewiesen werden. So erreichte das Vectran ${ }^{\circledR}-G a r n$ den höchsten Festigkeitszuwachs unter den untersuchten Garnen, wobei auch bei unverdrehtem Vectran ${ }^{\circledR}-G a r n$ eine Festigkeitssteigerung festgestellt werden konnte. Der Fixierprozess von Vectran ${ }^{\circledR}$ ging darüber hinaus mit einer leichten Zunahme der Dichte (unter einem Prozent) einher. Die Festigkeitszunahmen der beiden Garne sind Abbildung 8 dargestellt. in Abbildung 8
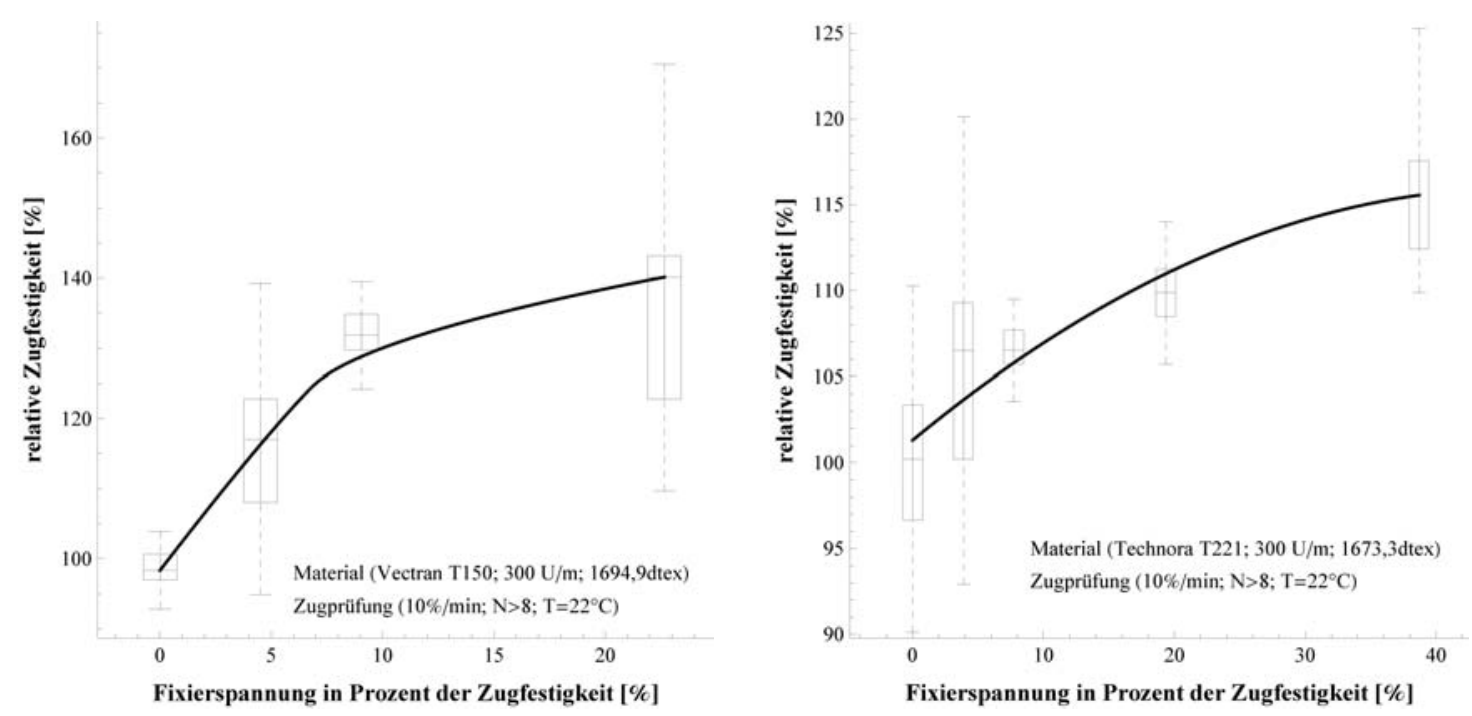


\section{Abbildung 8: Thermofixieren von Garnen: links Vectran ${ }^{\circledR}-$ Garn, rechts Technora ${ }^{\circledR}-G a r n$}

Im Anschluss an diese Untersuchungen wurden 6mm-Seilgeflechte thermofixiert. Hierzu wurden die Seile über das Halte-Reckwerk mit verschiedenen Fixierspannungen beaufschlagt und bei einer Vorschubgeschwindigkeit von $2 \mathrm{~m} / \mathrm{min}$ durch den $12 \mathrm{~m}$ langen Heiztunnel gefahren.

$A_{S}=\frac{T t}{\rho}$

$$
\text { Tt: Seilfeinheit [ktex]; } \rho \text { : Dichte }\left[\mathrm{g} / \mathrm{cm}^{3}\right]
$$

Die in den Diagrammen angegebenen Spannungen beziehen sich dabei auf den nach Gleichung 1 ermittelten Seilquerschnitt.

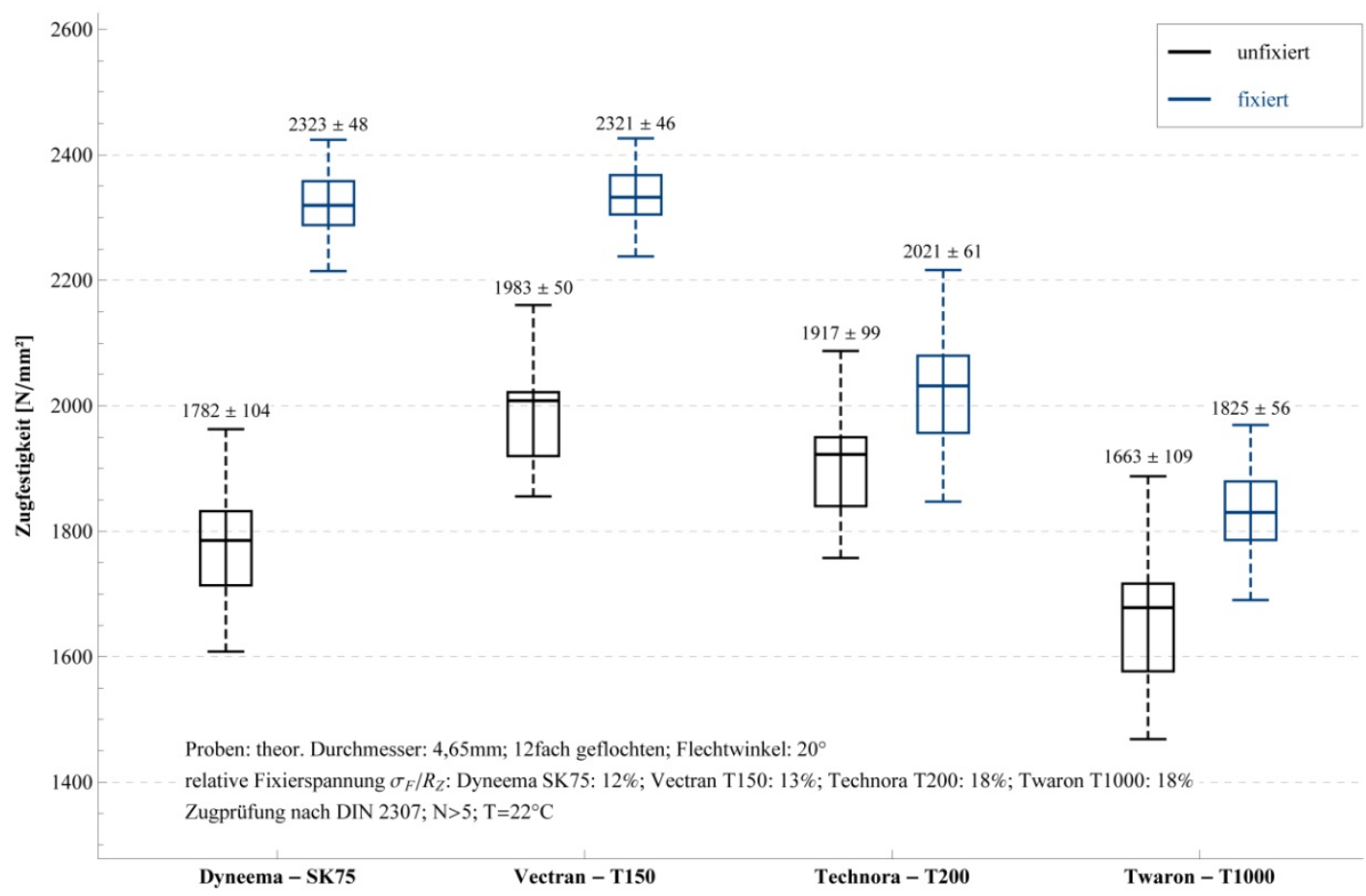

\section{Abbildung 9: Thermofixieren von 6mm-Seilgeflechten}

Abbildung 9 zeigt den Boxplot der Zugfestigkeiten unfixierter und fixierter $6 \mathrm{~mm}$-Seile. Anders als bei den Garnfestigkeiten erreichen hier die geflochtenen Dyneema ${ }^{\circledR}$-Seile die höchsten Festigkeitszuwächse. Dies lässt auf eine geringere Querempfindlichkeit im Vergleich zu den Vectran ${ }^{\circledR}$-Fasern schließen, da im Unterschied zum Verdrehen, höhere lokale Flächenpressungen, durch Überkreuzung der Litzen, entstehen. Konnte die Zugfestigkeit fixierter Technora ${ }^{\circledR}$-Seile aufgrund der Knickempfindlichkeit nur wenig gesteigert werden, so haben sie dennoch mit den anderen Seilen gemeinsam, dass das nach der t-Verteilung bestimmte Streufeld der ermittelten Zugfestigkeiten verringert werden konnte. Darüber hinaus zeigen die Spannungs/Dehnungsdiagramme der Seile einen deutlichen Rückgang der insbesondere in technischen Anwendungen nachteiligen Strukturdehnung und eine verminderte Energiedissipation (Hysteresekurve). Abbildung 10 zeigt beispielhaft das Spannungs-/Dehnungsverhalten der Technora ${ }^{\circledR}-$ Seile. Die Strukturdehnung konnte hier mit einer relativen Fixierspannung von $29 \%$ am stärksten minimiert werden, jedoch wies das Seil bereits äußerlich sichtbare Faserbrüche auf.

Weiterführende Untersuchungen an der TU Chemnitz beschäftigen sich mit der Biegewechselfestigkeit derartiger Seile. Es wird davon ausgegangen, dass durch die Erhöhung der Seildichte, verbunden mit einer höheren Formstabilität und einem homogeneren Kraftfluss, die Biegewechselfestigkeit thermofixierter Seile konstant bleibt oder sogar erhöht werden kann.

Erste Biegewechselversuche an einer Gegenbiegewechsel-Prüfmaschine konnten diese Annahme bestätigen. Abbildung 11 zeigt den Boxplot der Biegewechselzahlen unfixierter und fixierter 6mm- Dyneema ${ }^{\circledR}-$ Seile. Darin ist zu erkennen, dass die Biegewechselfestigkeit zunächst ansteigt, jedoch mit zunehmender Fixierspannung wieder abfällt. Die Ursache hierfür liegt in der zunehmenden Ausrichtung der Moleküle in Zugrichtung des 
Seiles begründet. Dadurch nimmt die, beim Lauf über Scheiben und Trommeln, notwendige Querfestigkeit des Seiles ab und führt zu einem schnelleren Versagen.

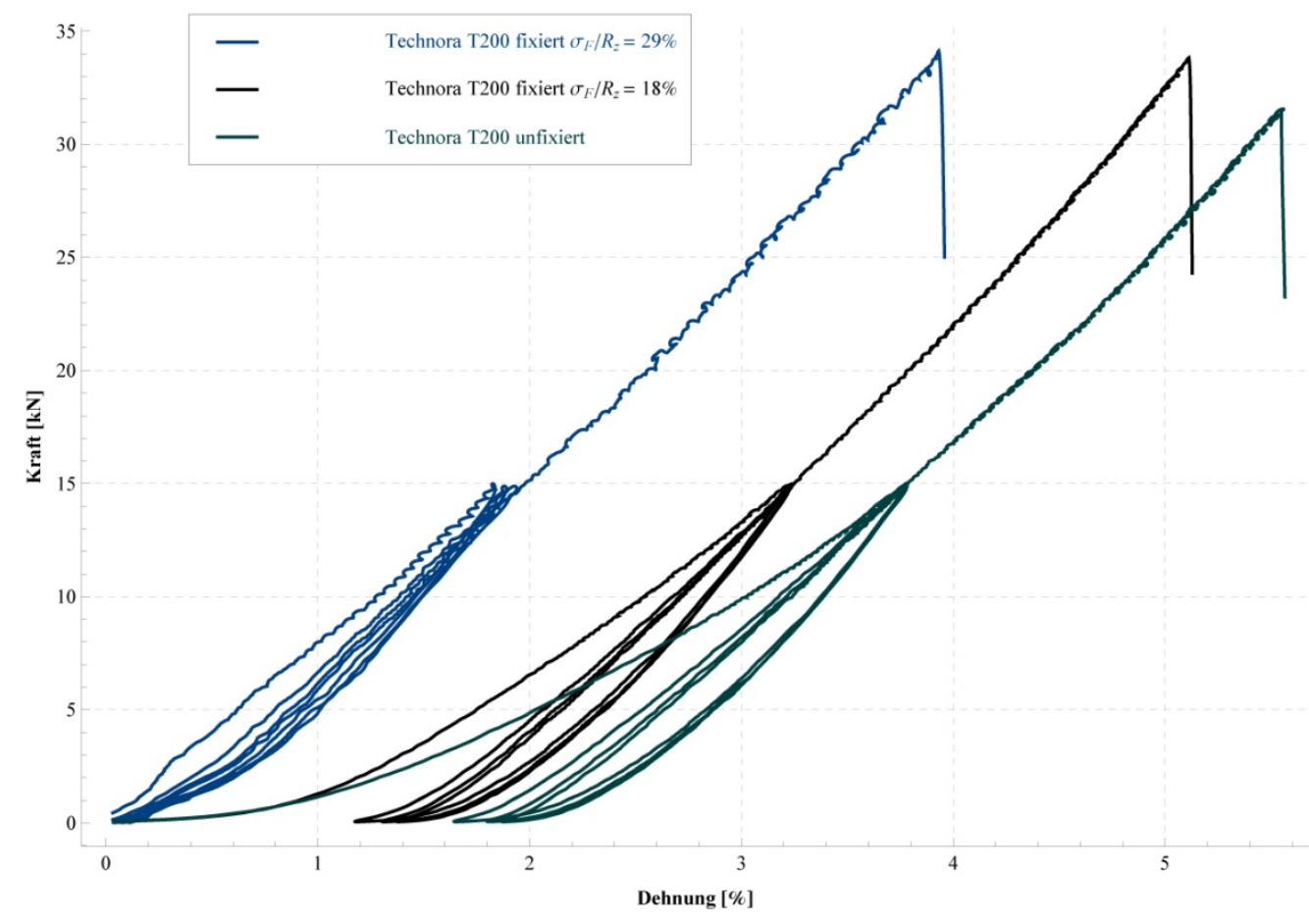

Abbildung 10: Strukturdehnung fixierter und unfixierter Seile 


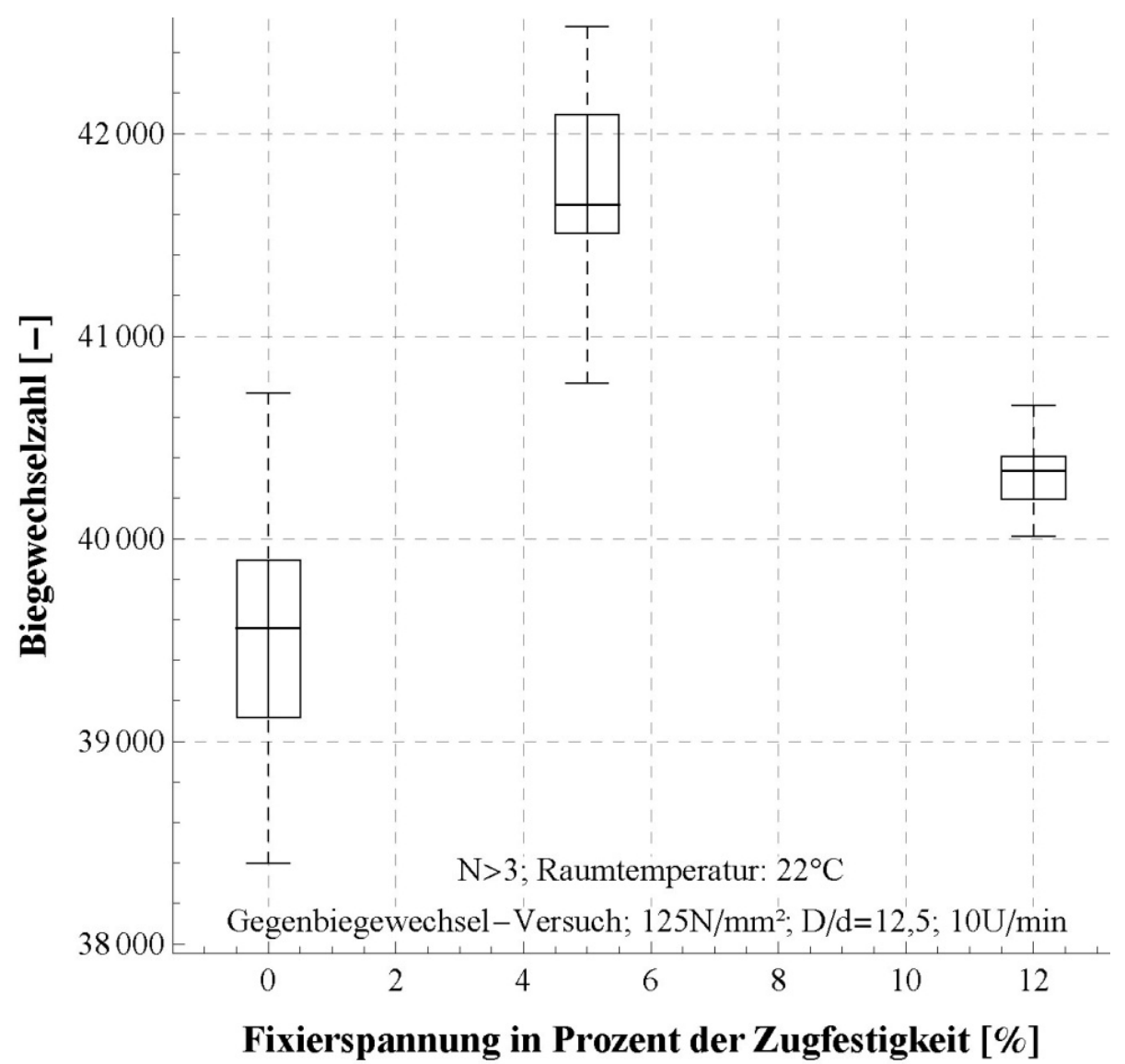

Abbildung 11: Biegewechselfestigkeit unfixierter und fixierter $6 \mathrm{~mm}$-Dyneema ${ }^{\circledR}$-Seile 


\section{Literatur}

[Ehr2003]

[Gle2005]

[Nen2006]

[Top1977]

[Sch2006]

[Kil1964]
Ehrenstein, G.W.; Riedel, G.; Trawiel, P.: Praxis der thermischen Analyse von Kunststoffen. München, Carl Hanser Verlag, 2003

Geo. Gleistein \& Sohn GmbH: Tauwerk für Industrie und Schifffahrt. Katalog, 2005 Nentwig, J.: Kunststoff-Folien. München, Carl Hanser Verlag, 2006

Topf, W.: Mischfreudige Chemiefasern-Herstellung und Qualität von Spinnfasermischgarnen. Lenzinger Berichte, Folge 42, S.173-S.182, 1977

Schenek, A.: Lexikon Garne und Zwirne. Frankfurt a.M., Deutscher Fachverlag, 2006

Kilby, W.F.: The mechanical properties of twisted continuous-filament yarns, TIT, Folge.55, Nr.12, 1964 